\title{
Effect of glycosylated vitamin D binding protein complexed with oleic acid on human myeloma and Hodgkin's lymphoma cultures
}

\author{
Jacopo J.V. Branca ${ }^{1 *}$, Rodney J Smith ${ }^{2}$, Emma Ward $^{2}$ and Marco Ruggiero ${ }^{3}$ \\ ${ }^{1}$ Department of Experimental and Clinical Medicine, University of Firenze, Italy \\ ${ }^{2}$ Macro Innovations Ltd, UK \\ ${ }^{3}$ Immuno Biotech Ltd, GBG
}

\begin{abstract}
$\mathrm{N}$-Acetylgalactosamine-glycosylated vitamin D binding protein (GalNAc-DBP) is known to stimulate macrophages and to directly inhibit angiogenesis and cancer cell growth in vitro. In this study, we evaluated the direct effect of GalNAc-DBP complexed with oleic acid on human multiple myeloma and Hodgkin's lymphoma cell lines (KMS-12-BM and L540 respectively) as well as the effect, on the same cell lines, of human spleen macrophages (CRL9853) previously activated by GalNAc-DBP complexed with oleic acid.

Cell viability and living cell number were evaluated respectively by Tetrazolium dye cell viability assay and by Trypan Blue staining. Interactions between activated macrophages and cancer cells were studied by time lapse photography. Our results show that GalNAc-DBP complexed with oleic acid inhibits the proliferation of myeloma and Hodgkin's lymphoma cells in a dose-dependent manner. Furthermore, GalNAc-DBP complexed with oleic acid activates human spleen macrophages which in turn phagocytise cancer cells. In addition, we observed that GalNAc-DBP complexed with oleic acid activates human spleen macrophages in vivo as documented by color-doppler ultrasonography of the spleen. These results demonstrate that GalNAc-DBP complexed with oleic acid has a double effect on myeloma and Hodgkin's lymphoma cancer cells: a direct inhibition of their proliferation and viability and, at the same time, an efficient macrophage activation leading to a significant depletion of cancer cell population. Taken together, these results suggest that GalNAc-DBP complexed with oleic acid may prove effective in the integrative immunotherapy of multiple myeloma and Hodgkin's lymphoma.
\end{abstract}

\begin{abstract}
Abbreviations: DBP: Vitamin D Binding Protein, FBS: Foetal Bovine Serum, Gal: Galactose, GalNAc-DBP: N-AcetylgalactosamineGlycosylated Vitamin D Binding Protein, GalNAc: N-Acetylgalactosamine, IDD: Intrinsically Disordered Domains, MAF: Macrophage Activating Factor, NO: Nitric Oxide, Sia: Sialic Acid

\section{Introduction}

In recent years the interest in the effects of vitamin $\mathrm{D}$ and other nutritional factors such as oleic acid in the integrative treatment of multiple myeloma and Hodgkin's lymphoma has increased [1]. Thus, these cancers are considered calcium and vitamin D sensitive, and it has been suggested that combined supplementation may be required for optimal chemoprevention [2]. Oleic acid, on the other hand, has been known for centuries for its anti-cancer properties and, according to a recent review "The beneficial effects of oleic acid in cancer processes can no longer be doubted" [3].
\end{abstract}

It has been known since 1988 that oleic acid is non-covalently bound to the vitamin D binding protein (DBP) that constitutes one of its carrier proteins in plasma and other biological fluids [4]. We recently elucidated the molecular interactions between oleic acid, vitamin $\mathrm{D}, \mathrm{DBP}$ and the vitamin $\mathrm{D}$ receptor [5]. In this context, it is worth noting that the anti-cancer effects of oleic acid are mediated by its interaction with proteins that are found in biological fluids such as milk or plasma [6]. Therefore, it can be hypothesized that DBP, by virtue of its complexing with oleic acid, may play a role in anti-cancer immune-surveillance that goes beyond that of a carrier protein.
DBP is encoded by a gene located on 4q12-q13 (HGNC: 4187), and it is also known as group-specific component (GC). DBP exists in different isoforms and the one designated Gc1s is glycosylated and comprises a linear O-linked trisaccharide of the type GalNAcGal-Sia attached to the threonine residue at position 420 [7]. During immune responses and in milk fermentation, sialic acid and galactose are enzymatically removed, and the Gc1s isoform of DBP with the remaining GalNAc covalently bound to threonine 420 is known to stimulate macrophages [8].

In addition to this effect on macrophage function, GalNAc-DBP, sometimes also designated DBP-MAF by some Authors (Macrophage Activating Factor) is known to directly inhibit tumour angiogenesis and human prostate and breast cancer cell proliferation in vitro [9-11].

Consistent with these observations, we previously demonstrated that GalNAc-DBP stimulated Raw 264.7 cells (a murine macrophage cell line) that were observed in vitro to phagocytise MCF-7 cells (human

Correspondence to: Jacopo J.V. Branca, Department of Experimental and Clinical Medicine, University of Firenze, 50134 Firenze, Italy, Tel: +39055 2758067; E-mail: jacopo.branca@libero.it

Key words: Hodgkin's lymphoma, macrophages, multiple myeloma, oleic acid, spleen, vitamin $D$

Received: February 24, 2015; Accepted: March 14, 2015; Published: March 17, 2015 
breast carcinoma cells) [5]. In those studies, however, GalNAc-DBP was used as an isolated, purified protein that is non-complexed with oleic acid as it occurs in physiological conditions. In addition, those results were obtained in a co-culture of activated murine macrophages and human breast cancer cells, and thus they could not be directly extrapolated to the conditions occurring in human neoplasia.

Therefore, we decided to study the effects of GalNAc-DBP complexed with oleic acid on human multiple myeloma (KMS-12BM) and Hodgkin's lymphoma (L540) cells as well as the effect, on the same cell lines, of human spleen macrophages (CRL9853) previously activated by GalNAc-DBP complexed with oleic acid.

We also studied the activation of spleen macrophages in vivo taking advantage of the recently published observation that macrophages activated by GalNAc-DBP complexed with oleic acid release the vasodilating molecule nitric oxide (NO), thus allowing for direct visualization of increased blood flow in the spleen through colordoppler ultrasonography [12].

\section{Materials and methods}

\section{Cell lines}

KMS-12-BM; L540: Human multiple myeloma (KMS-12-BM), and human Hodgkin's lymphoma (L540) cell lines were purchased from DSMZ (German Collection of Microorganisms and Cell Cultures, Braunschweig, Germany), and cultured in RPMI 1640 supplemented with $10 \%$ FBS and $2 \mathrm{mM}$ L-Glutamine (Life Technologies, Paisley, UK). Cultures were passaged every 3-4 days.

CRL9853: Human spleen macrophage was purchased from ATCC (American Type Culture Collection, Teddington, UK) and routinely cultured in IMDM supplemented with 10\% FBS and $2 \mathrm{mM}$ L-Glutamine (Life Technologies, Paisley, UK). Cultures were passaged every 3-4 days. Prior to assay, CRL9853 cells were activated culturing them for $72 \mathrm{~h}$ in the presence of GalNAc-DBP complexed with oleic acid at a concentration $100 \mathrm{ng} / \mathrm{mL}$ in complete medium.

\section{Stimuli}

GalNAc-DBP complexed with oleic acid, commercially available, was prepared at Immuno Biotech Ltd (Guernsey, Channel Island, GBG) with a procedure previously described [12]. A the end of the preparation, GalNAc-DBP complexed with oleic acid was analyzed at Macro Innovations Laboratories (Cambridge, UK) as described in Table 1.

\section{Cell proliferation assay}

Cell proliferation and viability were evaluated by the reduction of a Tetrazolium salt (WST-8) as an index of cell dehydrogenases' activity. KMS-12-BM and L540 cells were seeded into a 96 wells plate at a density of $3 \times 10^{4}$ cells/well in their appropriate starvation medium (without FBS). After incubation for $24 \mathrm{~h}$ the cell lines were treated for $24 \mathrm{~h}$ with the following different concentrations of GalNAc-DBP complexed with oleic acid [8-80-800 pM]. At the end of the treatment, the medium was replaced with $100 \mu \mathrm{l}$ of fresh starvation medium plus $10 \mu \mathrm{l}$ of WST -8 . The 96 wells plate was incubated for $3 \mathrm{~h}$ at $37^{\circ} \mathrm{C}$ and the optical density (O.D.) was directly measured at $\mathrm{A}_{450 \mathrm{~nm}}$ by Multiscan FC photometer (Thermo Scientific, Milano, Italy).

\section{Cell counting - Trypan Blue assay}

To corroborate the results obtained by the cell proliferation and viability assay, a viable cell count was performed. Briefly, KMS-12$\mathrm{BM}$ and L540 cells were plated into 6 wells plate at a density of $2 \times 10^{5}$ cells/well in starvation medium. After $24 \mathrm{~h}$ incubation, cells were treated with GalNAc-DBP complexed with oleic acid at the increasing concentrations [ $8-80-800 \mathrm{pM}$ ] for $24 \mathrm{~h}$. At the end of the treatment, a volume of cell suspension was collected and the viable cell number was counted by Trypan Blue staining.

\section{Video time-lapse photography}

KMS-12-BM and L540 cells were seeded into a 24 wells plate at a density of $1 \times 10^{6}$ cells/well with a $1 \mathrm{~mL}$ volume. The cells were allowed to settle for a minimum of $2 \mathrm{~h}$ prior to the addition of the CRL9853 macrophages that had been previously activated by GalNAc-DBP complexed with oleic acid. The staging mat was set at a temperature of $37^{\circ} \mathrm{C}$ and allowed to equilibrate prior to placement of the 24 wells plate (Figure 1).

The activated CRL9853 human spleen macrophages were added to a final concentration of $5 \times 10^{5}$ cells/well in $1 \mathrm{~mL}$. HEPES (Fisher Scientific, Loughborough, UK) was added to each well to provide a final concentration of $25 \mathrm{mM}$ to stabilize the culture $\mathrm{pH}$.

Once activated macrophages and the HEPES were added, the 24 wells plate was observed microscopically and an image selected. An initial frame was taken and a time-lapse film initiated. A frame was taken every 3 min until filming was stopped.

Table 1. Summary of Analysis.

\begin{tabular}{|c|c|c|}
\hline Test & Specification & Result \\
\hline Identification of Bulk Product Confirmed by anti-DBP Western Blot & Pass & Pass \\
\hline Identification of Bulk product by Non-Reduced Silver Stain & Presence of $44 \mathrm{kDa}$ band & Presence of $44 \mathrm{kDa}$ band \\
\hline Identification of Bulk Product by Non-Reduced Lectin Binding Western Blot & Detection at $44 \mathrm{kDa}$ & Detection at $44 \mathrm{kDa}$ \\
\hline Sterility by Membrane Filtration to meet EU and USP Pharmacopeia & No growth observed in samples tested after 14 days incubation & Pass \\
\hline Endotoxin on Final Filled Product & $<0.25$ & 0.05 \\
\hline pH of Final Filled Product & $\mathrm{pH} 7.0-7.9$ & $\mathrm{pH} 7.4$ \\
\hline Appearance of Final Filled Product & Clear colourless liquid & Clear colourless liquid \\
\hline Human Source Plasma Screened for HbsAG & Non-Reactive & Non-Reactive \\
\hline Human Source Plasma Screened for anti-HCV & Non-Reactive & Non-Reactive \\
\hline Human Source Plasma Screened for anti-HBc & Non-Reactive & Non-Reactive \\
\hline Human Source Plasma Screened for anti-HIV1 & Negative & Negative \\
\hline Human Source Plasma Screened for anti-HIV2 & Negative & Negative \\
\hline $\begin{array}{l}\text { Identification of Purified Source Material Confirmed by anti-DBP Western } \\
\text { Blot }\end{array}$ & Pass & Pass \\
\hline Purity of Purified Source Material by Coomassie stained Gel (visual) & $>95 \%$ & $>95 \%$ \\
\hline
\end{tabular}


A

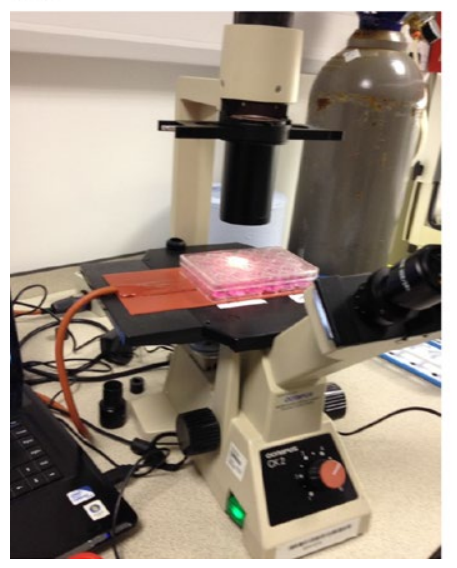

B

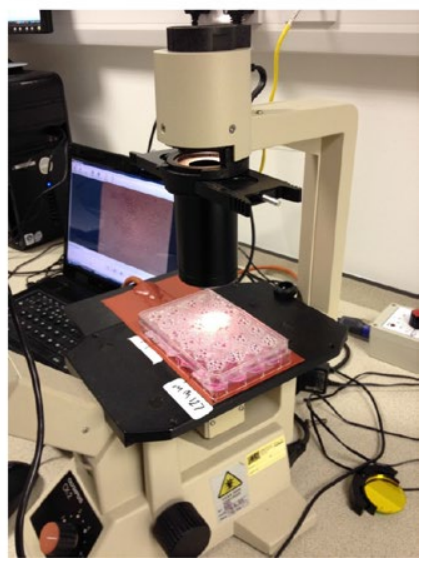

Figure 1. Video-time lapse photography setting at Macro Innovations Laboratories.

A

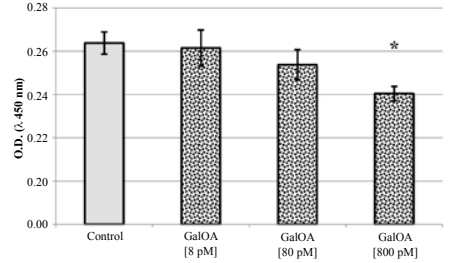

B

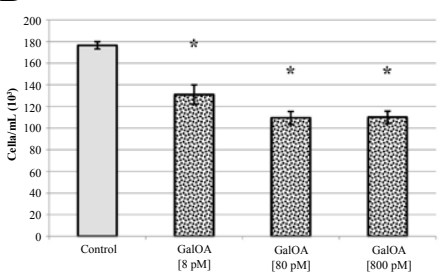

Figure 2. KMS-12-BM cell proliferation assay and Trypan Blue viable cell counts.

Panel A. GalNAc-DBP complexed with oleic acid is abbreviated in GalOA. Results for $[8 \mathrm{pM}]$ demonstrate a slight decrease on cell proliferation which becomes significantly greater at $[800 \mathrm{pM}]$ indicating a direct effect on cell proliferation. Panel B. The decrease in viable cell number as the GalNAc-DBP complexed with oleic acid concentration increases corroborates the observed cell viability assay data. Results are expressed as mean value \pm S.E.M. for five replicates. $\left({ }^{*} \mathrm{p}<0.01\right)$

Study of the effects of GalNAc-DBP complexed with oleic acid on human spleen macrophages in vivo

Macrophage activation in vivo was assessed by monitoring a volunteer's (MR) splenic blood flow before and after administration of GalNAc-DBP complexed with oleic acid. Thus, it is well known that activated macrophages release NO, a compound that causes vasodilatation and seems to be responsible for some of the anti-cancer properties of activated macrophages $[13,14]$. We studied the splenic blood flow with an ultrasound system (MyLab25Gold, Esaote, Genova, Italy) using the color-doppler technique before and after administration of GalNAc-DBP complexed with oleic acid (440-880 ng dissolved in 5 $\mathrm{mL}$ saline).

\section{Results}

\section{Cell proliferation assay}

Cell proliferation and viability of KMS-12-BM human multiple myeloma cells (Figure 2), evaluated both by Tetrazolium dye cell assay (Figure 2A) and by Trypan Blue staining (Figure 2B), decreased when the cells were treated with increasing concentrations of GalNAcDBP complexed with oleic acid. In particular, when cells were treated with GalNAc-DBP complexed with oleic acid [800 pM] a significant reduction $(\mathrm{p}<0.01)$ in cell viability was observed in comparison to the untreated control cells.

Qualitatively identical results were obtained with L540 human Hodgkin's lymphoma cells (Figure 3, panels A and B). Inhibition of cell proliferation and viability by GalNAc-DBP complexed with oleic acid was even more pronounced than that observed in human multiple myeloma cells.

\section{Video time-lapse photography}

Figure 4, shows selected frame shots over time of KMS-12-BM cells in the presence of CRL9853 human spleen macrophages that had been previously activated by GalNAc-DBP complexed with oleic acid under the conditions described in "Materials and Methods" and shown in Figure 1. Panel A, shows KMS-12-BM suspension culture typical growth pattern. Panel B, shows KMS-12-BM cells $24 \mathrm{~h}$ post addition of activated macrophages. CRL9853 spleen macrophages cultures appear as large masses of suspension cells and the dense collection of cells can be observed sitting on the KMS-12-BM suspension cells. The activated macrophages are extremely motile under observation and move around clearing the KMS-12-BM cells. During the phagocytosis, smaller dense "clusters" of cells break off and scavenge the KMS-12-BM cells quite

A

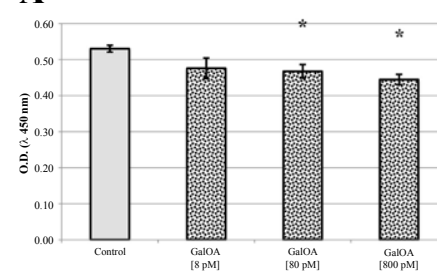

B

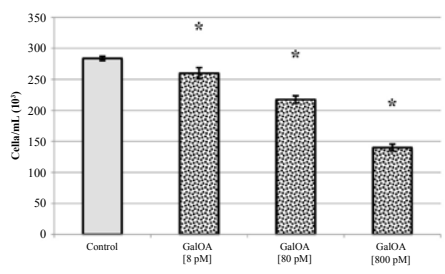

Figure 3. L540 cell proliferation assay and Trypan Blue viable cell counts.

Panel A. GalNAc-DBP complexed with oleic acid is abbreviated in GalOA. Results for $[8 \mathrm{pM}]$ demonstrate a slight decrease on cell proliferation which becomes significantly greater at $[800 \mathrm{pM}]$ indicating a direct effect on cell proliferation. Panel B. The decrease in viable cell number as the GalNAc-DBP complexed with oleic acid concentration increases corroborates the observed cell viability assay data. Results are expressed as mean value \pm S.E.M. for five replicates. $(* \mathrm{p}<0.01)$.

A

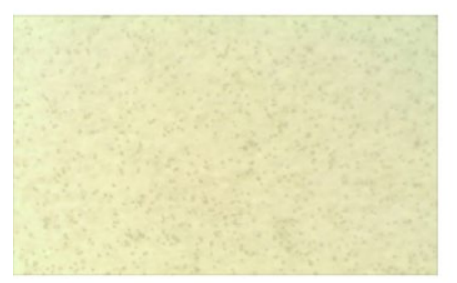

B

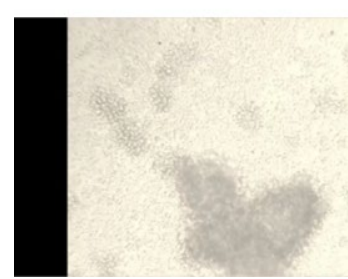

Figure 4. Video time-lapse photography of KMS-12-BM challenged with human spleen macrophages that had been previously activated by GalNAc-DBP complexed with oleic acid.

Selected frame shots over time of KMS-12-BM cells in the presence of CRL9853 human spleen macrophages that had been previously activated by GalNAc-DBP complexed with oleic acid under the conditions described in "Materials and Methods". For the interpretation of the images; please see the "Results" section. 
rapidly. Panel C, shows KMS-12-BM cells $48 \mathrm{~h}$ post addition of activated human macrophages. CRL9853 cells now have a few large cell mass "clusters" and many smaller "clusters" as can be observed (red arrows). The number of KMS-12-BM cells has been decreased and the clearance of cells can be easily observed. Finally, panel D, shows KMS-12-BM cells $72 \mathrm{~h}$ post addition of activated human macrophages CRL9853. Activated CRL9853 cells are still extremely active with the phagocytic activity and are clearing the KMS-12-BM cells from the culture. This image shows the smaller clusters of cells as indicated by the red arrows. The clusters have become smaller and rapid moving seeking out and destroying the remaining KMS-12-BM cells. Qualitatively identical results were obtained with L540 human Hodgkin's lymphoma cells (not shown).

\section{Ultrasonography of the spleen and activation of spleen macrophages in vivo}

Since the results described above indicate that human spleen macrophages were activated by GalNAc-DBP complexed with oleic acid in vitro, we decided to study whether a comparable activation could be observed in vivo. To this end, we exploited the fact that activated macrophages release NO [13]. Considering the impressive effects of $\mathrm{NO}$ in pre-clinical models of cancer where it slows tumour growth and enhances the efficacy of both chemotherapy and radiotherapy [15], we reasoned that the observation of activation of spleen macrophages in vivo could further corroborate the hypothesis of proposing GalNAcDBP complexed with oleic acid as an integrative immunotherapeutic agent.

Therefore, we opted to study the variation of the splenic blood flow, following the administration of GalNAc-DBP complexed with oleic acid ( $880 \mathrm{ng}$, dissolved in $5 \mathrm{~mL}$ saline) by nebulisation. According to our hypothesis, GalNAc-DBP complexed with oleic acidwould be absorbed into the bloodstream and it would stimulate the huge amount of macrophages residing in the spleen [16]. Therefore, we would expect a significant release of $\mathrm{NO}$ by activated macrophages in this organ, with consequent vasodilation and increase of blood flow that we could measure by color-doppler ultrasonography.

One of us (MR), self-administered GalNAc-DBP complexed with oleic acid ( $880 \mathrm{ng}$, dissolved in $5 \mathrm{~mL}$ saline) by nebulisation for about 5 min. After that, the subject, who is an expert radiologist, self-examined his spleen with color-doppler ultrasonography.

Figure 5, panels A and B, shows the ultrasonographic appearance of the spleen of the volunteer before nebulisation; morphology, size and ultrasonographic structure appear normal. The splenic blood flow could be appreciated in correspondence of the hilum of the spleen. The mean diameter of the blood vessels at the hilum was $4.4 \mathrm{~mm}$. Figure 5, panels $\mathrm{C}$ and $\mathrm{D}$, shows the splenic blood flow $15 \mathrm{~min}$ after nebulisation. The vasodilation and the increase in blood flow can be appreciated. The mean diameter of the blood vessels at the hilum was $6.1 \mathrm{~mm}$. Postulating a regular geometry of the blood vessels as if they were perfectly circular, the increase in the section area could be calculated in about $85 \%$. We interpreted this vasodilation and increase in blood flow as a consequence of intra-splenic macrophage activation with the release of NO that in turn was responsible for hilar blood vessel dilation. This effect lasted for, at least, $48 \mathrm{~h}$.

\section{Discussion}

GalNAc-DBP complexed with oleic acid can be considered a physiologic/natural compound that is produced in mammal plasma
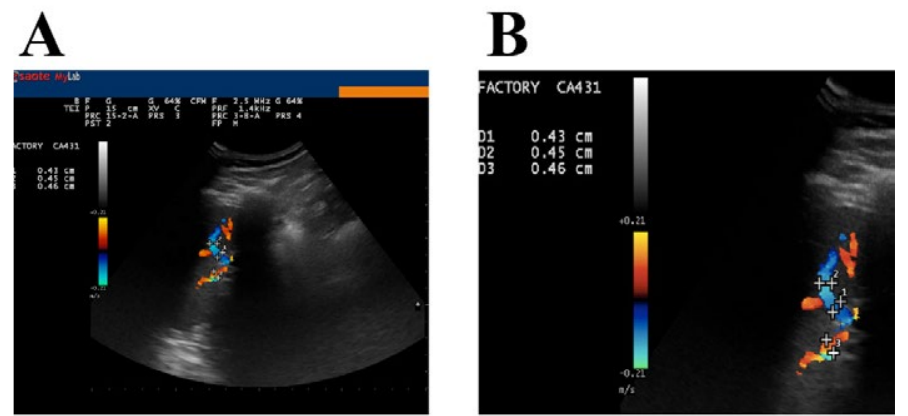

C

D
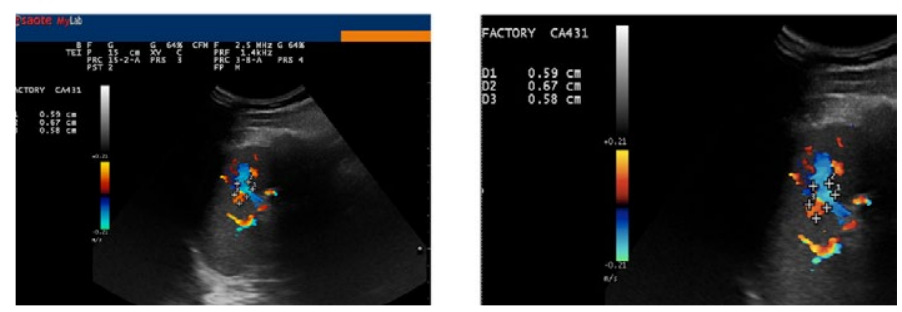

Figure 5. Ultrasonographic appearance of the spleen before and after administration of GalNAc-DBP complexed with oleic acid.

Panel A: Morphology, size and ultrasonographic structure of the spleen appear normal: splenic blood flow can be appreciated in correspondence of the hilum of the spleen. Panel B (magnification): The mean diameter of the blood vessels at the hilum was 4.4 $\mathrm{mm}$. Measurements were taken during the exam using the proprietary application of the ultrasound system. Panels $C$ and $D$ (magnification): Splenic blood flow $15 \mathrm{~min}$ after nebulization of GalNAc-DBP complexed with oleic acid. The mean diameter of the blood vessels at the hilum was $6.1 \mathrm{~mm}$.

during immune responses [17] and in milk and colostrum during fermentation [18,19]. GalNAc-DBP, as an isolated protein, has been studied in a number of experimental models of diseases, from osteopetrosis [17] to cancer [10] and autism [20].

However, the effects of GalNAc-DBP complexed with oleic acid have never been studied in controlled experimental models. In other words, all the studies on this subject concentrated on the effects of the isolated protein and not on the overall effects of the physiological assembly of the protein with oleic acid. Therefore, in the context of a proposable integrative immunotherapeutic approach, we decided to study the effects of GalNAc-DBP complexed with its natural counterpart that is oleic acid.

In this study, we demonstrate that GalNAc-DBP complexed with oleic acid, stimulated human spleen macrophages, both in vitro and in vivo although the demonstration of macrophage activation in vivo was indirect. The effects of the complex GalNAc-DBP-oleic acid were significantly more pronounced when compared to the effect of GalNAc-DBP alone; oleic acid per se did not stimulate human spleen macrophages (not shown).

Human spleen macrophages that had been pre-activated by GalNAc-DBP complexed with oleic acid were able to recognize and phagocytise human cancer cells i.e. multiple myeloma and Hodgkin's lymphoma cells.

However, GalNAc-DBP complexed with oleic acid, in addition to this effect involving activation of tumoricidal macrophages, also exerted a direct effect on human myeloma and Hodgkin's lymphoma cells cultures, and it inhibited their proliferation and viability. These results are consistent with those previously reported by Gregory 
et al. and by Pacini et al. on human prostate and breast cancer cells respectively $[10,11]$.

We hypothesize that the multitude of biological effects exerted by this protein when associated with oleic acid can be explained by the recent discovery of the so-called "Intrinsically Disordered Domains" (IDDs). It is now widely recognized that proteins with IDDs are found in organisms from all kingdoms of life and, in eukaryotes, they perform a wide range of biological functions, including regulation and signaling [21]. These domains lack a fixed or ordered three-dimensional structure and cover a spectrum of states from fully unstructured to partially structured that may include random coils, molten globules, and large multiple domains connected by flexible linkers. Their mobile flexibility and structural instability are encoded by their amino acid sequences [22].

Thus, IDDs are characterized by the presence of hydrophobic amino acids interspersed with positively and negatively charged amino acids. Therefore these domains cannot bury sufficient hydrophobic core to fold like stable globular proteins [23]. However, when a bulky hydrophobic moiety, such as the molecule of oleic acid, binds to a protein region where an IDD is present, the molecular configuration of the IDD changes dramatically together with the specificity of interaction with other target molecules.

Using disorder prediction algorithms based on primary sequence composition, we identified two IDDs in the GalNAc-DBP (Figure 6) and, interestingly, one of these IDD, designated IDD2 because of its position in the amino acid sequence, is in the same region where oleic acid binds. The shallow hydrophobic cleft where oleic acid binds is located between domains II and III, which is between positions 288 and 371, thus encompassing the IDD2 that is located between positions 298-325.

Therefore, we hypothesize that the multifarious effects of this protein, in particular when it is complexed with its natural counterpart that is oleic acid, are due to the presence of this peculiar IDD2 modified

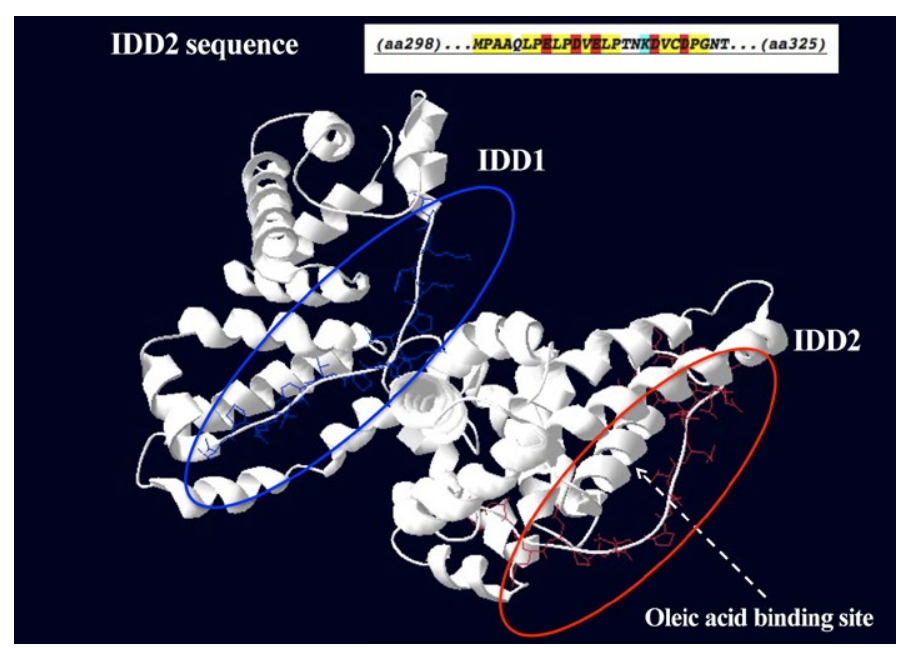

Figure 6. Proposed three-dimensional structure of GalNAc-DBP with the two Intrinsically Disordered Domains.

The two Intrinsically Disordered Domains (IDD) are evidenced in blue (IDD1) and in red (IDD2). The characteristics of the amino acids in the sequence of the IDD2 (amino acids 298-325) are depicted in the insert. In yellow, hydrophobic amino acids. In red, negatively charged amino acids. In light blue, lysine, a positively charged amino acid. The shallow hydrophobic cleft where oleic acid binds is indicated by the dotted arrow. The fatty acid binding site is located between domains II and III, which is between positions 288 and 371, thus encompassing the IDD2. by the non-covalent binding with a hydrophobic molecule such as oleic acid.

This finding could be of particular interest in the context of an integrated immunotherapy of cancer based on molecular structures. For example, one of the major human oncogenes, $m y c$, encodes an intrinsically disordered protein that can be bound and inhibited by small molecules through formation of dynamic complexes [21].

Therefore, it can be hypothesized that GalNAc-DBP complexed with oleic acid binds Myc (and/or other products of oncogenes or tumour suppressor genes), thus counteracting the effects of the oncoprotein at the molecular level. The presence of positively and negatively charged amino acids in the proximity of the oleic acid binding renders the molecular complex highly dynamic, thus favouring the interaction with multiple target proteins, such as Myc or p53, that exhibit similar IDDs [24].

\section{Conflict of interest}

MR was the external consulting scientific director of Immuno Biotech, Ltd (the company isolating and purifying the $\mathrm{N}$-Acetylgalactosamine-glycosylated vitamin $\mathrm{D}$ binding protein and producing the Oleic Acid complexes) until February 2015. However, MR had no prior knowledge of the experimental protocols and he did not participate in the actual planning and execution of the in vitro experiments described in this paper. He participated in the analysis of the results and in the molecular modelling only after the results had been verified and validated by the other Authors as well as in the ultrasonography studies.

\section{References}

1. Schwartz GG, Skinner HG (2007) Vitamin D status and cancer: new insights. Curr Opin Clin Nutr Metab Care 10: 6-11. [Crossref]

2. Peterlik M, Grant WB, Cross HS (2009) Calcium, vitamin D and cancer. Anticancer Res 29: 3687-3698. [Crossref]

3. Carrillo C, Cavia Mdel M, Alonso-Torre SR (2012) Antitumor effect of oleic acid mechanisms of action: a review. Nutr Hosp 27: 1860-1865. [Crossref]

4. Williams MH, Van Alstyne EL, Galbraith RM (1988) Evidence of a novel association of unsaturated fatty acids with Gc (vitamin D-binding protein). Biochem Biophys Res Commun 153: 1019-1024. [Crossref]

5. Thyer L, Ward E, Smith R, Fiore MG, Magherini S, et al. (2013) A novel role for a major component of the vitamin $\mathrm{D}$ axis: vitamin $\mathrm{D}$ binding protein-derived macrophage activating factor induces human breast cancer cell apoptosis through stimulation of macrophages. Nutrients 5: 2577-2589. [Crossref]

6. Nakamura T, Aizawa T, Kariya R, Okada S, Demura M, et al. (2013) Molecular mechanisms of the cytotoxicity of human $\alpha$-lactalbumin made lethal to tumor cells (HAMLET) and other protein-oleic acid complexes. J Biol Chem 288: 14408-14416. [Crossref]

7. Borges CR, Jarvis JW, Oran PE, Nelson RW (2008) Population studies of Vitamin D Binding Protein microheterogeneity by mass spectrometry lead to characterization of its genotype-dependent O-glycosylation patterns. J Proteome Res 7: 4143-4153. [Crossref]

8. Ravnsborg T, Olsen DT, Thysen AH, Christiansen M, Houen G, et al. (2010) The glycosylation and characterization of the candidate Gc macrophage activating factor Biochim Biophys Acta 1804: 909-917. [Crossref]

9. Kisker O, Onizuka S, Becker CM, Fannon M, Flynn E, et al. (2003) Vitamin D binding protein-macrophage activating factor (DBP-maf) inhibits angiogenesis and tumor growth in mice. Neoplasia 5: 32-40. [Crossref]

10. Gregory KJ, Zhao B, Bielenberg DR, Dridi S, Wu J, et al. (2010) Vitamin D binding protein-macrophage activating factor directly inhibits proliferation, migration, and uPAR expression of prostate cancer cells. PLoS One 5: e13428. [Crossref] 
11. Pacini S, Punzi T, Morucci G, Gulisano M, Ruggiero M (2012) Effects of vitamin D-binding protein-derived macrophage-activating factor on human breast cancer cells. Anticancer Res 32: 45-52. [Crossref]

12. Ruggiero M, Ward E, Smith R, Branca JJ, Noakes D, et al. (2014) Oleic Acid, deglycosylated vitamin D-binding protein, nitric oxide: a molecular triad made lethal to cancer. Anticancer Res 34: 3569-3578. [Crossref]

13. Stuehr DJ, Nathan CF (1989) Nitric oxide. A macrophage product responsible for cytostasis and respiratory inhibition in tumor target cells. $J$ Exp Med 169: 1543-1555. [Crossref]

14. Nathan CF, Hibbs JB Jr (1991) Role of nitric oxide synthesis in macrophage antimicrobial activity. Curr Opin Immunol 3: 65-70. [Crossref]

15. Singh S, Gupta AK (2011) Nitric oxide: role in tumour biology and iNOS/NO-based anticancer therapies. Cancer Chemother Pharmacol 67: 1211-1224. [Crossref]

16. Swirski FK, Nahrendorf M, Etzrodt M, Wildgruber M, Cortez-Retamozo V, et al. (2009) Identification of splenic reservoir monocytes and their deployment to inflammatory sites. Science 325: 612-616. [Crossref]

17. Schneider GB, Benis KA, Flay NW, Ireland RA, Popoff SN (1995) Effects of vitamin D binding protein-macrophage activating factor (DBP-MAF) infusion on bone resorption in two osteopetrotic mutations. Bone 16: 657-662. [Crossref]

18. Hollis BW, Draper HH (1979) A comparative study of vitamin D binding globulins in milk. Comp Biochem Physiol B 64: 41-46. [Crossref]

19. Gomme PT, Bertolini J (2004) Therapeutic potential of vitamin D-binding protein. Trends Biotechnol 22: 340-345. [Crossref]

20. Siniscalco D, Bradstreet JJ, Cirillo A, Antonucci N (2014) The in vitro GcMAF effects on endocannabinoid system transcriptionomics, receptor formation, and cell activity of autism-derived macrophages. J Neuroinflammation 11: 78. [Crossref]

21. Follis AV, Galea CA, Kriwacki RW (2012) Intrinsic protein flexibility in regulation of cell proliferation: advantages for signaling and opportunities for novel therapeutics. Adv Exp Med Biol 725: 27-49. [Crossref]

22. Oldfield CJ, Dunker AK (2014) Intrinsically disordered proteins and intrinsically disordered protein regions. Annu Rev Biochem 83: 553-584. [Crossref]

23. Gibbs EB, Showalter SA (2015) Quantitative biophysical characterization of intrinsically disordered proteins. Biochemistry 54: 1314-1326. [Crossref]

24. Joerger AC, Fersht AR (2010) The tumor suppressor p53: from structures to drug discovery. Cold Spring Harb Perspect Biol 2: a000919. [Crossref]

Copyright: (C) 2015 Branca JJV. This is an open-access article distributed under the terms of the Creative Commons Attribution License, which permits unrestricted use, distribution, and reproduction in any medium, provided the original author and source are credited. 